\title{
2D Fourier Analysis and its Application to Study of Scaling Properties and Fractal Dimensions of $\varepsilon$-Martensite Distribution in $\gamma$-Matrix of Fe-Mn-Si Alloy
}

\author{
A.Yu. Pasko, A.A. Likhachev, Yu.N. Koval and V.I. Kolomytsev \\ Institute of Metal Physics, Phase Transformation Department, Nat. Acad. Sc. Ukr., Vernadsky St. 36, \\ 252142 Kiev, Ukraine
}

\begin{abstract}
D Fourier analysis is proposed to obtain quantitative information on the structural amplitudes in reciprocal space for different heterophase systems. An original technique for profound computer processing of optical images transformed into an appropriate digital form is developed. Correlation Fourier analysis is applied $t$ ) study a spatial distribution of $\gamma \rightarrow \varepsilon$ martensitic transformation product in Fe-Mn-Si shape memory alloy. All evident spatial self-similarity and scaling behavior is observed, indicating a fractal-like distribution of $\varepsilon$-phase in $\gamma^{-}$ matrix. The structural factor versus wave number dependence shows three scaling invariant regions with different scaling dimensions and crossover-regions between them. A simple fractal model is proposed to interpret experimental results.
\end{abstract}

\section{INTRODUCTION}

Modern investigations of martensitic transformation kinetics in different metallic shape memory and othe: SMART materials, and their applications in advanced technologies require adequate development of ne $\%$ analysis and control methods for the multi-phase microstructural distribution of transformation product; and its evolution under external thermal and mechanical driving forces. Traditionally these problems arie solved by the ordinary statistical metallography or X-ray small angle scattering technique. In addition to these methods, a two-dimensional (2D) Fourier analysis is proposed in the present paper to obtain quantitative information on the heterophase system structural amplitudes in reciprocal wave space. An original program for direct computer processing of corresponding optical images transformed into an appropriate digital form is used.

Correlation Fourier analysis is applied to study a spatial distribution of $\gamma \rightarrow \varepsilon$ martensiti: transformation product in Fe-Mn-Si shape memory alloy (SMA). Optical micrographs obtained by Maki and Tsuzaki [1] are used as source images. Like other ferrous-based SMA such as Fe-Ni, reported earlie; by Hornbogen [2], this one also shows an evident spatial self-similarity and scaling behavior, indicating a fractal-like distribution of $\varepsilon$-phase in $\gamma$-matrix. The wave number dependence of the structural factor reveals three scaling invariant regions with different scaling dimensions and crossover regions betwe'"l them. A simple fractal model is proposed to interpret experimental results.

\section{STRUCTURAL FACTOR SCALING PROPERTIES OF HETEROPHASE SYSTEM}

Let us introduce the main objects and their quantitative definitions that will be used in the present study. The first one is a microscopic quantity that can be obtained from experiment and is defined as the local density of the. second phase in parent matrix $\sigma(\mathbf{x})$ taking unit value $\sigma(\mathbf{x})=1$ everywhere in the area occupied by the second phase and zero value $\sigma(x)=0$ in the parent phase region. Similar spin-like variables contain the most detailed information about $2 \mathrm{D}$ heterophase microstructure images that can be obtained by using common physical methods such as the optical metallography or transmission electron microscopy. Naturally, these microscopic quantities are very irregular ones, and include both regulc.: information and randomly fluctuating characteristics. As a result, reasonable physical information can to: 
usually obtained from the basic spatially averaged sequence of multi-point products of the microscopic local density field $\sigma(\mathbf{x})$. These are known as multi-point correlation functions. Most of physical applications are mainly restricted to a study of the second order correlation functions.

Following a similar scheme, one can define single- and two-point correlation functions

$$
S^{(1)}=\frac{1}{V} \int_{V} d^{2} \mathbf{x} d(\mathbf{x})=z_{s}, \quad S^{(2)}(\mathbf{x})=\frac{1}{V} \int_{V} d^{2} \mathbf{x}^{\prime} d\left(\mathbf{x}^{\prime}\right) d\left(\mathbf{x}^{\prime}+\mathbf{x}\right)
$$

Here a spatial averaging procedure is carried out over $2 \mathrm{D}$ image cross-section $V$, and $\mathbf{x}$ is $2 \mathrm{D}$ spatial coordinate vector. The single-point average $S^{(1)}$ gives the volume fraction $z_{s}$ of the second phase, while $S^{(2)}(\mathbf{x})$ represents so-called two-point correlation function of microscopic local density variations or, in other words, the structural factor of a given heterophase system. The structural function must satisfy some obvious rules such as

$$
S^{(2)}(0)=\frac{1}{V} \int_{V} d^{2} \mathbf{x} \sigma^{2}(\mathbf{x})=z_{s}
$$

following directly from its definition and spin-like variables algebra rule $\sigma^{2}(\mathbf{x})=\sigma(\mathbf{x})$.

$2 \mathrm{D}$ Fourier transform of characteristic function $\sigma(\mathbf{x})$ can be written as

$$
\left.\sigma_{\mathbf{k}}=\frac{1}{V} \int_{V} d^{2} \mathbf{x} \alpha \mathbf{x}\right) \exp (-i \mathbf{k x})
$$

It is known from different physical applications that the structural function Fourier image

$$
S_{F}(\mathbf{k})=\frac{1}{V} \int_{V} d^{2} \mathbf{x} S^{(2)}(\mathbf{x}) \exp (-i \mathbf{k} \mathbf{x})=\left|\sigma_{\mathbf{k}}\right|^{2}
$$

usually determines the power spectrum of density fluctuations. In our case the structural factor contains information on the power spectrum of the second phase density distribution in heterophase system.

There are many physical objects showing scaling behavior in the wide range of scales lower than a correlation distance $r_{c}$ and larger than some microscopic scale $\rho$. This means that the structural function obeys a power law:

$$
\begin{gathered}
S^{(2)}\left(\mathbf{x} ; \rho, r_{c}\right) \propto|\mathbf{x}|^{-\mu}, \quad \rho \ll|\mathbf{x}| \ll<r_{c} \\
S_{F}\left(\mathbf{k} ; \rho, r_{c}\right) \propto|\mathbf{k}|^{-\mu_{F}}, \quad r_{c}^{-1} \ll|\mathbf{k}| \ll<\rho^{-1}
\end{gathered}
$$

The scaling dimension indexes $\mu$ and $\mu_{F}$ take usually non-integer values. Such objects are often classified as the fractals, and this type of behavior can be attributed to the hypothesis of general scaling similarity for structural factor with respect to a simultaneous rescaling transformation of variables $\mathbf{x}$ and $\rho$ :

$$
S^{(2)}(\xi \mathbf{x}, \xi \dot{\rho})=\xi^{-\mu} S^{(2)}(\mathbf{x}, \rho)
$$

where $\mathbf{x}$ and $\rho$ are expressed in the units of correlation scale $r_{c}$. Putting $\xi=|\mathbf{x}|^{-1}$ or $\xi=\rho^{-1}$, one can easily obtain two important rules following directly from the scaling invariance hypothesis (7):

$$
S^{(2)}(|\mathbf{x}|, \rho)=|\mathbf{x}|^{-\mu} S^{(2)}(1, \rho /|\mathbf{x}|)=\rho^{-\mu} S^{(2)}(|\mathbf{x}| / \rho, 1)
$$

As a result, we find

$$
\begin{gathered}
S^{(2)}(|\mathbf{x}|, \rho) \rightarrow|\mathbf{x}|^{-\mu} S^{(2)}(1,0), \quad \rho<<|\mathbf{x}| \ll<1 \\
S^{(2)}(|\mathbf{x}|, \rho) \rightarrow \rho^{-\mu} S^{(2)}(0,1), \quad|\mathbf{x}| \ll<\rho
\end{gathered}
$$

Further we will consider two types of fractal objects: 1) fractal "areas" with topological dimension $D_{T}=2$; and 2) fractal "curves" with topological dimension $D_{T}=1$. The total specific areas $z_{s}$ (for $D_{T}=2$ ) or specific lengths $l_{s}$ (for $D_{T}=1$ ) of similar fractal objects is known to be a definite power function of microscopic characteristic scale $\rho$ in accordance with the following formulae:

$$
z_{s}(\rho) \propto \rho^{2-D_{s}}, \quad l_{s} \propto \rho^{1-D_{s}}
$$


where $D_{S}$ is the fractal dimension of these objects. On the other hand, according to equation (2), the structural correlation function must satisfy a normalization rule

$$
S^{(2)}(0, \rho)= \begin{cases}z_{s}(\rho), & D_{T}=2 \\ l_{s}(\rho), & D_{T}=1\end{cases}
$$

By the way, taking into account the consequence of scaling formula (10)

$$
S^{(2)}(0, \rho)=\rho^{-\mu} S^{(2)}(0,1)
$$

one can obtain an important correspondence rule between the scaling dimension $\mu$ of structural correlation function, fractal dimension $D_{S}$ and topological dimension $D_{T}$ of a given fractal object:

$$
\mu=D_{S}-D_{T}
$$

Similarly, the corresponding rule for the Fourier power spectrum scaling dimension can be found

$$
\mu_{F}=2-\mu=2+D_{T}-D_{S}
$$

These relationships give a necessary basis for correct interpretation of $2 \mathrm{D}$ Fourier analysis results and fractal dimensions for different types of objects under study.

The most well known and widely investigated scale-invariant behavior is associated with the systems undergoing different types of phase transitions near their critical points [3-6]. The spinodal decomposition is the next traditional object for scaling examination. During the last time the list of fractal objects has been essentially extended to include a great number of non-traditional areas of applications [7-11]. Thus, it has been recently found that the heterophase structure formed as a result of martensitic transformation in some SMA has evidently fractal-like character $[2,12,13]$.

\section{FOURIER ANALYSIS PROCEDURE, APPLICATION AND RESULTS}

Practical realization of the proposed approach to statistical correlation analysis of heterophase microstructures has some specific features due to incompleteness of source information. In fact, the characteristic function obtained from experiment is usually represented by a sample on a finite set of points resulted from the procedure of rasterization of a small part of entire picture. Nevertheless, general Fourier transform formulae can still be used in this case. For this purpose one should consider the data matrix as it were continued periodically from an appropriate observation area over the whole plane. As a result, the discreteness and periodicity of the source function lead respectively to the periodicity and discreteness of corresponding Fourier image, and we come to the well-known discrete Fourier transform expressions:

$$
\begin{gathered}
\sigma_{\mathbf{k}}=\frac{1}{\sqrt{N}} \sum_{\mathbf{x} \in A} \sigma(\mathbf{x}) \exp (-i \mathbf{k x}) \\
S^{(2)}(\mathbf{x})=\frac{1}{\sqrt{N}} \sum_{\mathbf{k} \in B}\left|\sigma_{\mathbf{k}}\right|^{2} \exp (i \mathbf{k x})
\end{gathered}
$$

Here the function $\sigma(\mathbf{x})$ representing a given heterophase configuration is defined on a square $2 \mathrm{D}$ lattice in a rectangular area $A$ containing $N=p \times q$ points, and the wave vector $\mathbf{k}$ takes values on the corresponding rectangular lattice inside the square Brillouin zone $B$ of reciprocal space.

To investigate a system for scaling properties one can verify whether the power spectrum of this system characteristic function obeys some power law (6). It is also convenient to analyze the power spectrum averaged over all the direction of wave vector as a function of its magnitude. Since the wave vector takes only discrete values, a preliminary interpolation of function $S_{F}(\mathbf{k})$ is required. We use a simple square interpolation between lattice points. To smooth the resulting curve some more in numerical calculations, it is reasonable to perform an averaging procedure by integrating the interpolated function over rings of finite width. The Brillouin zone is to be partitioned into a set of $n$ rings bounded by radii $k_{0}, \ldots, k_{n}$, then the averaged power spectrum can be represented by the series 


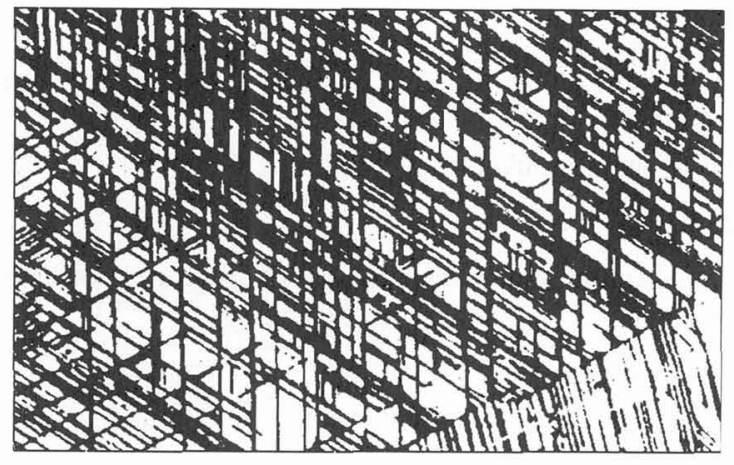

a)

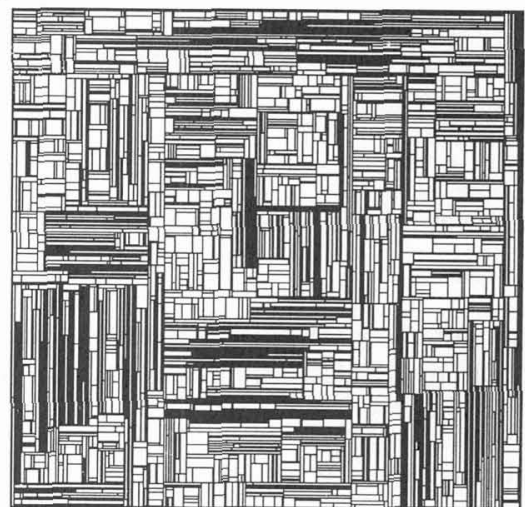

D)

Figure 1: Two-color images of martensitic microstructure in Fe-Mn-Si alloy (a) and model martensitic system (b).

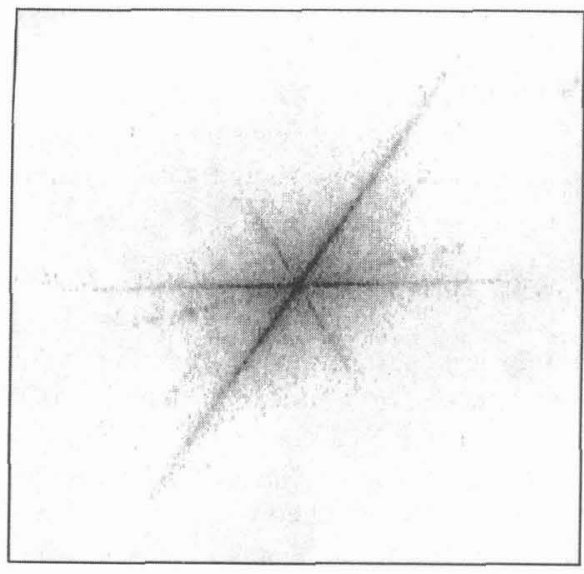

a)

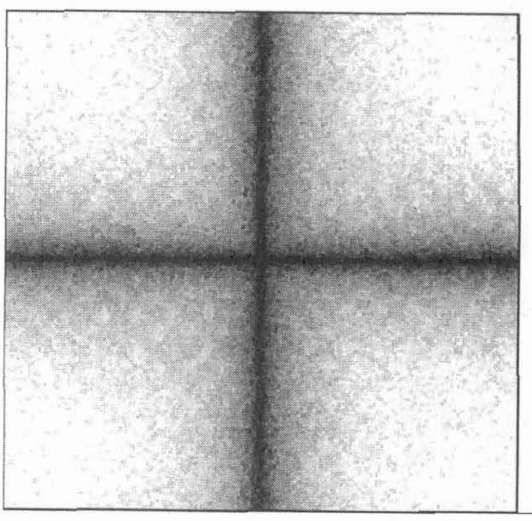

ס)

Figure 2: Fourier spectrum for martensitic microstructure (a) and model system (b).

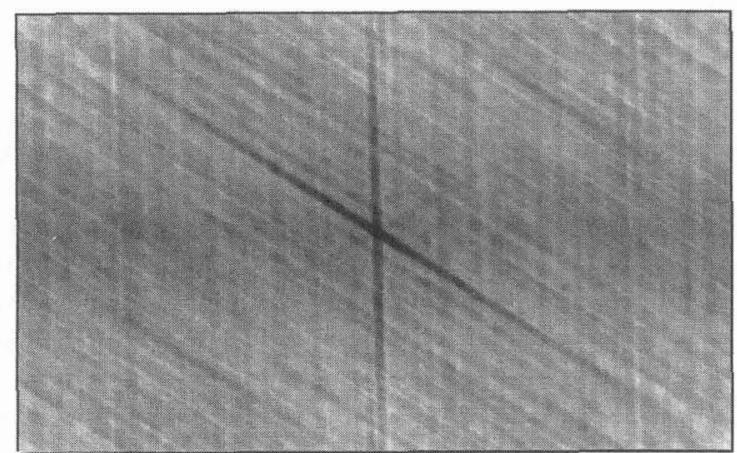

a)

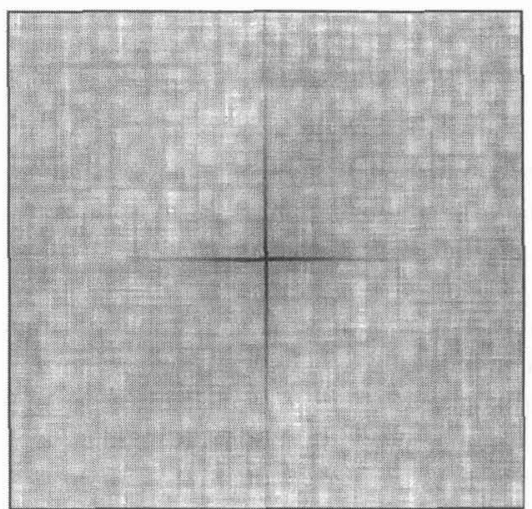

b)

Figure 3: Correlation function for martensitic microstructure (a) and model system (b). 


$$
S_{F}\left(\left(k_{i-1}+k_{i}\right) / 2\right)=\frac{1}{\pi\left(k_{i}^{2}-k_{i-1}^{2}\right)} \int_{k_{i-1} \leq|\mathbf{k}| k_{i}} d^{2} \mathbf{k} S_{F}(\mathbf{k}), \quad i=1, \ldots, n
$$

For scaling analysis a well-known graphical method is used. As follows from formula (6), for fractal objects the power spectrum versus wave vector magnitude dependence plotted in logarithmic axes will look as a straight line with inclination determined by the scaling index. In practice, we should build a similar graph for function (18) and analyze its shape looking for the intervals of scaling behavior. Complex systems frequently have several such regions with different scaling indexes corresponding to different objects or phenomena. These indexes are determined separately for each interval by a standard meansquare approximation routine.

The procedure of computer Fourier analysis includes several sequential steps. Source data can be obtained from original micrographs of heterophase structure using a scanner or another device for input of optical images into computer. The data must be in one of the standard graphics formats. This raw image usually does not conform to the spin-like form of characteristic function $\sigma(\mathbf{x})$, contains many details related to other factors, and therefore needs additional processing. Refined picture must be black and white, so that the area occupied by martensitic crystals is depicted, for example, in black, while the parent phase matrix displays as white background. Fig. 1(a) presents such picture obtained from the optical micrograph of heterophase structure formed in consequence of $\gamma \rightarrow \varepsilon$ martensitic transformation in Fe-Mn-Si alloy [1]

Then the specially developed computer program calculates the power spectrum $\left|\sigma_{k}\right|^{2}$ and correlation function $S^{(2)}(\mathbf{x})$ of the source image according to equations $(16,17)$, the fast Fourier transform (FFT) algorithm being used. Moreover, the power spectrum radial distribution (18) is computed. To visualize obtained images and make their analysis easier, the power spectrum and correlation function data can be converted to pictures. The colors may be chosen such that, for instance, white corresponds to the minimum value, and black symbolizes the maximum value of the data array. For Fig. 1(a) being taken as the source image, Fig. 2(a) shows the distribution of Fourier amplitude absolute value $\left|\sigma_{k}\right|$ in the Brillouin zone, and Fig. 3(a) displays the spatial correlation function. To improve perception of the built pictures a non-linear response curve and appropriate filtering were used. Fig. 4(a) illustrates the application of scaling analysis to the system concerned, for each scaling interval the corresponding scaling index is indicated.

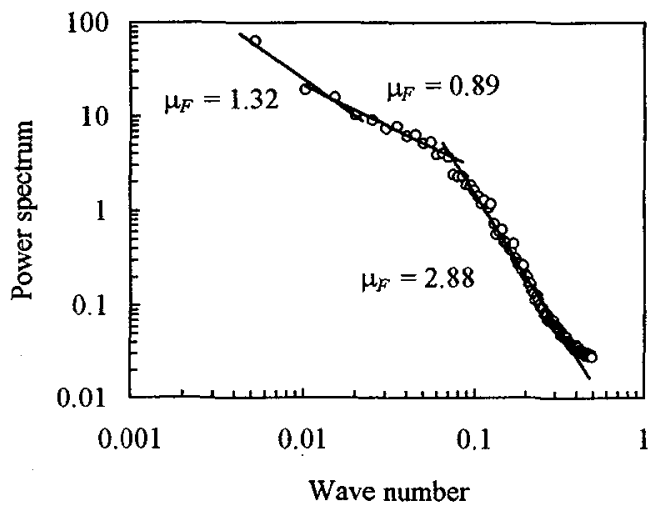

a)

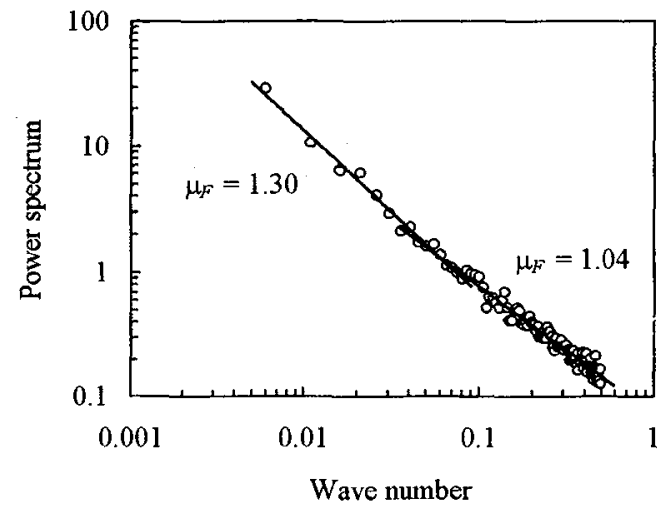

b)

Figure 4: Power spectrum radial distribution versus wave number for martensitic microstructure (a) and model system (b).

In the present paper we also propose and analyze a simple fractal model for microstructure formation during martensitic transformation. We follow an idea that the fractality and scaling behavior observed in SMA microstricture can be explained as a direct result of impossibility for each new martensitic crystal appeared at any stage of transformation to penetrate the other variant crystals formed before. Therefore the model algorithm is based on the sequence of thin crystals of two possible orientations growing 
subsequently from randomly distributed "embryo" points up to the intersection with the system of early formed crystals. The resulting microstructure containing about 3000 crystals is shown in Fig. 1(b). Correspondent Fourier spectrum, correlation function and power spectrum radial distribution are presented in Fig. 2(b), 3(b) and 4(b) respectively.

\section{DISCUSSION AND CONCLUSIONS}

Analyzing $\gamma+\varepsilon$ microstructure in Fe-Mn-Si alloy (Fig. 1(a)) and a two-phase structure simulated in the framework of proposed model (Fig. 1(b)), one can observe that martensitic crystals of different orientations form a completely connected area with isolated austenitic "windows" between them. The average scale of these windows is the first important microscopic scale of the system. The second microscopic scale associated with the thickness of martensitic $\varepsilon$-phase crystals is also well seen in Fig. 1(a) while the thickness scale of model crystals in Fig. 1(b) can be considered as infinitely small. As a result of such hierarchy of spatial scales, the corresponding radial Fourier power spectrum shows complex scaling behavior. In particular, $\gamma+\varepsilon$ system (Fig. 4(a)) displays three different regions: 1) a large scale region with $\mu_{F} \approx 1.3$ representing the range of scales between the picture size and window scale; 2 ) an intermediate region between the window and thickness scales with $\mu_{F} \approx 0.9$; and 3) a small scale region below this thickness scale with $\mu_{F} \approx 2.9$. Similar behavior, excluding small scale region, is observed for the model power spectrum (Fig. 4(b)). Only the large scale region with $\mu_{F} \approx 1.3$ can be interpreted as a result of impossibility for the crystals of different variants to penetrate each other, which is suggested in the model. The intermediate scaling dimension $\mu_{F} \approx 1$ is trivial and can be easily explained by a very small ratio between the thickness of crystals and window scale. Finally, the small scale region with $\mu_{F} \approx 2.9$ is related to the thickness distribution of martensitic crystals.

Visual representation of Fourier spectrum (Fig. 2(a,b)) and correlation function (Fig. 3(a,b)) contairis additional information on the angular distribution of corresponding quantities, which helps to determine the preference orientations of crystals in heterophase system. One can see a star-like figure in the center of each picture with rays being parallel (for correlation function images) or perpendicular (for Fourier spectrum ones) to the alignment directions of narrow crystals.

Thus, the special mathematical processing of microstructure images based on $2 \mathrm{D}$ Fourier analysis enables to obtain important information about statistical and fractal properties of heterophase systems.

\section{Acknowledgments}

This work was partially supported by INTAS grant No. 93-1202 ext.

\section{References}

[1] Maki T. and Tsuzaki K., "Transformation behavior of $\varepsilon$-martensite in Fe-Mn-Si shape memory alloys", Proc. Int. Conf. ICOMAT-92, Monterey, 20-24 July 1992, C.M. Wayman and J. Perkins Eds., pp. 1151-1162.

[2] Hornbogen E. Z. Metallk. 78 (1987) 352-354.

[3] Ahlers G., Kornblit A., Salamon M.B., Phys. Rev. B 9 (1974) 3932.

[4] Als-Nielsen J., Dietrich O., Phys. Rev. 153 (1966) 711.

[5] Kadanoff L.P., Phys. Rev. Lett. 34 (1975) 1005-1008.

[6] Wilson K.G., Kogut J., Phys. Rep. C 12 (1974) 75.

[7] Grossman T., Aharony A., J. Phys. A 20 (1987) L1193-L1201.

[8] Mandelbrot B.B., J. Stat. Phys. 34 (1983) 895-930.

[9] Mandelbrot B.B., Passoja D.E., Paullay A.J., Nature 308 (1984) 721-722.

[10] Meakin P., Phys. Rev. A 27 (1983) 1495-1507.

[11] Meakin P., Stanley H.E., Coniglio A., Witten T.A., Phys. Rev. A 32 (1985) 2364-2369.

[12] Jost N., Hornbogen E., Pract. Met. 25 (1988) 157-173.

[13] Kindo K., Hazumi K., Date M, J. Phys. Soc. Jap. 57 (1988) 715-717. 